\title{
Space Science and Technology Partnership Forum: Integration with Commercial In- Space Assembly Activities
}

\author{
Gregory Benjamin ${ }^{1}$ and Alejandro Pensado ${ }^{2}$ \\ Analytical Mechanics Associates, Inc., Hampton, VA, 23681, USA
Dale C. Arney, Ph.D. ${ }^{3}$, Sharon A. Jefferies ${ }^{4}$, Matthew A. Stafford ${ }^{5}$, Frederic H. Stillwagen ${ }^{6}$, and Phillip A. Williams, Ph.D. ${ }^{7}$
NASA Langley Research Center, Hampton, VA, 23681, USA \\ Erica Rodgers, Ph.D. ${ }^{8}$ \\ NASA Headquarters, Washington, DC, 20024, USA \\ Joseph Fulton ${ }^{9}$ \\ University of Maryland at College Park, College Park, MD, 20742, USA \\ Nicholas M. Houghton ${ }^{10}$ \\ Michigan State University, East Lansing, MI, 48824, USA \\ Alexander Mazarr ${ }^{11}$ \\ Virginia Polytechnic and State University, Blacksburg, VA, 24061, USA
}

\begin{abstract}
The interagency Space Science and Technology (S\&T) Partnership Forum was established in 2015 with participation from the United States Air Force, the National Aeronautics and Space Administration, and the National Reconnaissance Office. Seeking to leverage synergies and influence agency portfolios with a focus on key pervasive and game-changing technologies, the S\&T Partnership Forum successfully identified and prioritized several collaboration topic areas with high potential for future cross-agency work. The S\&T Partnership Forum determines the forum strategy, goals, and objectives, as well as the strategies and objectives specific to each collaboration topic area. In November 2018, the Partnership held a public open forum that focused on the topic area of in-space assembly
\end{abstract}

${ }^{1}$ Aerospace Concepts Engineer, AMA-Inc.

${ }^{2}$ Aerospace Concepts Engineer, AMA-Inc., AIAA Member.

${ }^{3}$ Capability and Technology Assessment Lead, Space Mission Analysis Branch, AIAA Member.

${ }^{4}$ Aerospace Engineer, Space Mission Analysis Branch, AIAA Senior Member.

${ }^{5}$ Aerospace Engineer, Space Mission Analysis Branch, AIAA Member.

${ }^{6}$ Aerospace Engineer, Space Mission Analysis Branch, AIAA Member.

${ }^{7}$ Senior Systems Analyst, Space Mission Analysis Branch.

${ }^{8}$ Senior Technologist, Office of Chief Technologist.

${ }^{9}$ Student Intern, Space Mission Analysis Branch.

${ }^{10}$ Student Intern, Space Mission Analysis Branch.

${ }^{11}$ Student Intern, Space Mission Analysis Branch. 
(iSA). This open forum was coordinated to facilitate government and commercial dialogue, collect data, and perform data analysis to identify potential cross-agency collaboration between government and commercial participants for in-space assembly and promising technologies. This paper discusses the analysis performed on the commercially provided data in relation to previously identified government needs, observations on the correlation between technologies and capabilities between government and commercial industry, and recommendations for future government collaborations with commercial industry for iSA.

\section{Introduction}

\section{A. Space Science and Technology Partnership Forum}

The Space Science and Technology (S\&T) Partnership Forum serves to coordinate and facilitate partner dialogue, perform data analysis, and assemble data products into recommendations for partnerships to be executed within the S\&T community at the program and project levels within the partnering agencies. The principal partners of the S\&T Partnership Forum are the United States Air Force (USAF), the National Reconnaissance Office (NRO), and the National Aeronautics and Space Administration (NASA), with additional agencies within the Department of Defense (DOD) (specifically, the U.S. Naval Research Laboratory (NRL) and the Defense Advanced Research Projects Agency (DARPA)) as affiliate partners. The meetings and activities of the S\&T Partnership Forum have resulted in the identification of specific areas among the participating agencies to explore the next steps in a possible cooperation endeavor.

The S \& T Facilitation and Analysis Team's work on the assessment of in-space assembly (iSA) as a collaboration topic area spans three phases, with each phase providing a measurable return on investment (ROI) aligned with the Partnership's goals. The phases also progressively built an understanding of the criteria for successful partnerships for interagency and commercial collaboration. Each phase consisted of four elements: (1) pre-work, (2) a technical interchange meeting (TIM), (3) analysis, and (4) production of deliverables. The TIM was central to each phase and was facilitated by the Facilitation and Analysis Team. At the TIM, representatives from the participating space agencies in the collaboration topic area of iSA set objectives, discussed needs, presented information or data, and made key decisions required for the goals of each phase.

As a follow up to the TIM-1 and TIM-2, held during Phases 1 and 2 respectively, the S\&T Partnership Forum held an Industry Open Forum (TIM-3) in November 2018. This open forum was coordinated to facilitate government and commercial dialogue, collect data, and perform data analysis to identify potential cross-agency collaborations between government and U.S. commercial companies for iSA. As a result, the participating government agencies were able to gather insight and knowledge of the iSA technologies currently being developed by these U.S. commercial companies. It is important to note, this paper does not intend to give a single technology and company as the right solution for future steps of iSA, but rather to provide general points of discussion and recommendations.

\section{Summary of Phase 1: Identifying the Value Proposition of iSA Interagency Collaboration}

In Phase 1, the Facilitation and Analysis Team worked to establish a value proposition and strategic framework for interagency collaboration in iSA within the partnership as a foundation to deliver value and achieve four iSA topic objectives [1]:

1) Establish baseline of government iSA work;

2) Collectively describe benefits of iSA architectures;

3) Establish value for partnering on iSA; and

4) Communicate and document TIM proceedings, baseline assessment, benefits, value proposition, and strategic plan.

2. Summary of Phase 2: U.S. Agency iSA Activities Analysis

Phase 2 collected and analyzed the partners' inputs to discover gaps, synergies, and redundancies in the capability needs and program efforts across the partner agencies. The S\&T Partnership identified 46 iSA capability needs within 14 capability areas of S\&T interagency governmental partner interest. The objectives for Phase 2 were:

1) Collect and prioritize iSA capability data and collect iSA demo platform data,

2) Perform government iSA capabilities analysis

3) Assess potential iSA demonstration platforms, and

4) Communicate results and make recommendations. 


\section{B. Phase 3 S\&T Partnership Industry Open Forum (TIM-3)}

Phase 3 examined the intersection of government and commercial objectives in iSA to identify potential U.S. commercial and government complementary roles. The Phase 3 TIM-3 was configured as an industry open forum. TIM-3 focused on current government activities in iSA, commercial iSA activities, capabilities, developments, and systems, and how the commercial systems and developments relate to iSA capability needs of government agencies within the S\&T Partnership.

During the open forum/TIM-3, government participants from the Air Force Research Lab (AFRL), USAF Space and Missile Systems Center (Air Force Space Command/SMC), NASA, and NRL presented to the audience of commercial companies the current work and status of iSA activities within their perspective agencies. Subsequently, there was a dialogue session between government and commercial participants.

After the Open Forum/TIM-3, the S\&T Facilitation and Analysis Team aggregated the responses from an online market research questionnaire they had distributed before the TIM and developed a dataset to analyze the responses. This paper focuses on the results of the Phase 3 analysis performed by the S\&T Facilitation and Analysis Team (Fig. 1), with an attempt to identify and align iSA capabilities across the government and industry arenas in which there are opportunities for collaboration and for a joint iSA effort amongst government agencies and industry. In addition, this paper presents the S\&T Facilitation and Analysis Team's key points from the Phase 3 analysis, as well as assessment findings and recommendations for the government.

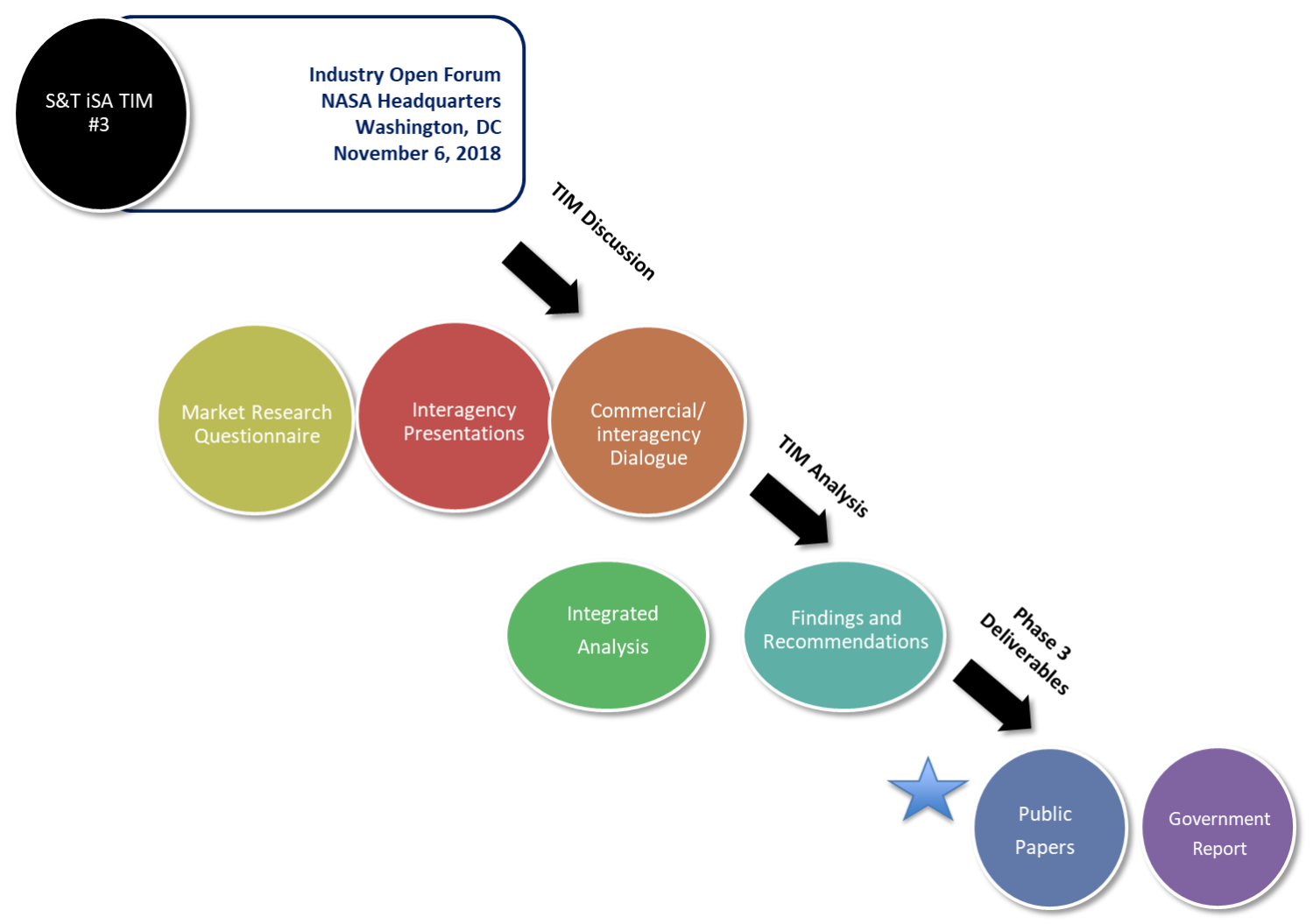

Fig. 1 Phase 3 iSA overall data collection and analysis flow performed in support of the S\&T Partnership Forum. 


\section{Data Collection}

The data collected from industry companies in this analysis comprise U.S.-owned commercial companies who completed the S\&T market research questionnaire.

\section{Market Research Questionnaire}

Prior to the Industry Open Forum/TIM-3 in November 2018, commercial companies were asked to complete a market research questionnaire that asked about their company's anticipated timeline for the availability of an iSA market; current iSA technologies, iSA systems and system capabilities; future space system(s) capabilities that capitalize on iSA; current iSA challenges/barriers that an industry/government partnership may help overcome; and the challenges/barriers their company foresees for future operational iSA systems.

Using the questionnaire responses as one branch of analysis, the Facilitation and Analysis Team was able to understand the current state of the commercial iSA capabilities, technologies, related systems, and how these systems and capability developments correspond to the iSA needs of the government partners for the companies in this assessment.

It is key to note that the market research questionnaire only asked for information directly related to iSA. While the S\&T Partnership Forum and Facilitation and Analysis Team recognize there are similarities between iSA and both in-space servicing (iSS) and in-space manufacturing (iSM), expanding this analysis to iSS and iSM was out of scope of the S\&T Partnership Forum's current assessment. Therefore, the questionnaires were sent to companies with servicing and manufacturing developments directly applicable to an iSA capability, and they were asked to submit information only about iSA, not iSS or iSM.

In addition to the market research questionnaire, there was dialogue between government space agencies and U.S.owned commercial companies about the technology and capability developments, as well as current activities commercial companies are doing in iSA, and how these activities and developments relate to the iSA capability needs of the government agencies within the S\&T partnership.

\section{E. Commercial Data set}

The data consisted of commercial companies who completed the $S \& T$ market research questionnaire. The majority of companies that submitted responses have connections to U.S. government space agencies; therefore, the information in this paper likely represents government-influenced industry trends. The data collection methods used do not provide sufficient information to confidently differentiate the government's influence from purely private sector activities.

Further, from the company's description of their overall iSA goals or vision, the S\&T analysis team was able to categorize each company into a specific market area. Below are the company market areas:

Satellites \& Space Structures - The focus area of a company's iSA technology is to have the capability of being able to assemble or "put together" spacecraft's, satellites, modular platforms, and/or large space structures.

Satellite Servicing - The focus area of a company's iSA technology is to have the capability of being able to service other satellites through advanced robotics and tools.

Robotics - The focus area of a company's iSA technology is to be able to use robots to perform in-space construction and/or manipulation with precision and efficiency.

Satellite Manufacturing - The focus area of a company's iSA technology is to have the capability of being able to produce satellite components in space or from component space resources (in situ).

Interfaces - The focus area of a company's iSA technology is to have the capability of being able to actually put space components together. Moreover, the physical action of connecting the space components together.

Additive Manufacturing - The focus area of a company's iSA technology is to have the capability of being able to print materials in space and join these materials to be assembled together in space.

Large Telescope - The focus area of a company's iSA technology is to have the capability of being able to use iSA to build and operate large telescopes in space

Software, iSA - The focus area of a company's iSA technology to have the capability of being able to use operating systems, utilities, programs, and applications to allow for robotic/autonomous manipulation, procedures, and path planning for iSA.

\section{Industry's Perspective on iSA}

This section presents the responses collected in which companies were asked about the 46 iSA capabilities of interagency governmental interest [1]. The companies were asked whether they were pursuing or planning to pursue the ability to provide each capability within the next 15 years, and if so, when the development was projected to be 
ready. The goal of this effort was to understand what industry is working on, which capability area(s) industry activity is leaning towards, and what their current projected timelines are.

\section{A. Industry Activity}

Companies were asked if they were currently planning or pursuing developments that could provide each of the iSA capabilities within the next 15 years (Appendix, Table 7). Although information was provided through written responses on the specific technologies that the companies were working on, this information was not consistent enough to map back to individual capabilities. Therefore, the results presented are only the information collected at the capability level, and do not encompass the varying technologies that can be applied to each capability, nor do they capture the Technology Readiness Levels (TRL) of their development. The results are compiled in Fig. 2. In Fig. 2, "Capability ID" refers to the S\&T iSA capability identification from the previous analysis [1]. To see the "Capability ID's" and coresponding titles, please refer to the Appendix.

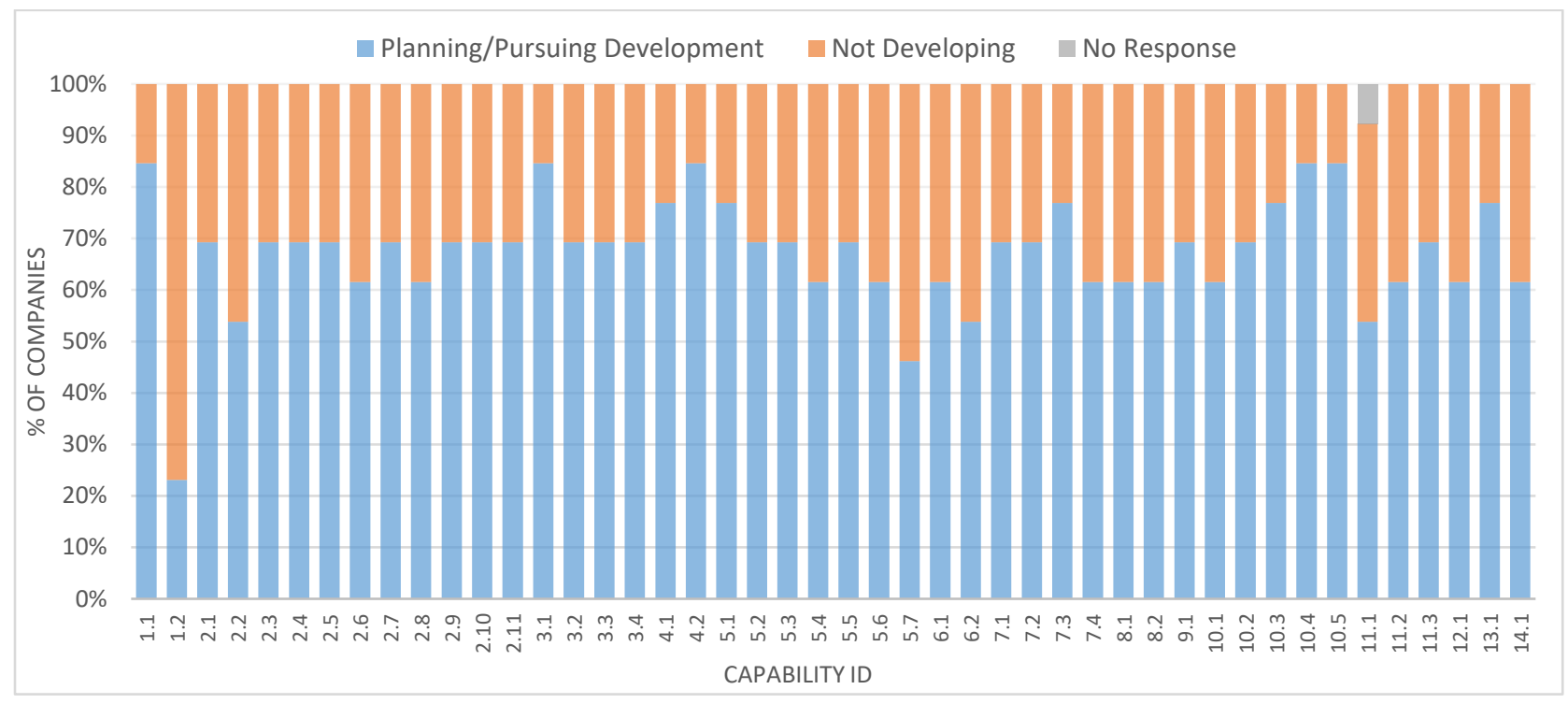

Fig. 2 The compilation of the responses of capabilities industry is planning to pursue or are currently pursuing that could be provided within the next 15 years.

Commercial respondents reported are actively pursuing each S \& T Forum iSA capability, which maybe either an indication of government and industry activities aligning or an indication that the companies surveyed may be pursuing these capabilities because of pre-existing collaborations with government. There is a visible outlier where Capability 1.2 (inflatable systems) has only $23 \%$ of companies planning to or currently pursuing that capability. Most capabilities fall within a standard deviation of the average.

Each company was allowed to add any capability they were working on that was not part of the 46 identified capabilities. A few entries were submitted but are not presented in this figure, as they were not capabilities relevant to iSA and were not scored by everyone.

It can be noted that Capability 5.7 (quantitative performance predictions for autonomous systems) also has a large number of companies not pursuing that capability. Capabilities 2.2 (long reach manipulation) and 6.2 (standard but secure communication protocols to accommodate interaction with other (TBD) associated systems) have an equal percent of companies that do not have plans to pursue versus are actively planning or are pursuing the capability. Although there were no financial data collected, Fig. 2 can give a general idea of where the interest and investment reside for the surveyed companies. Table 1 list the six capabilities that respondents most frequently reported they are pursuing or planning to pursueFig. 1Error! Reference source not found.. It is key to note that there are six capabilities listed in Table 1 due to the fact that five of the six capabilities are the same percentage. 
Table 1 Sorted six most frequent capabilities industry respondents are pursuing and planning to pursue that will be developed within 15 years.

\begin{tabular}{|c|l|c|}
\hline $\begin{array}{c}\text { Capability } \\
\text { ID }\end{array}$ & \multicolumn{1}{|c|}{ Description } & $\begin{array}{c}\text { Percent of } \\
\text { Companies } \\
\text { Pursuing }\end{array}$ \\
\hline 10.4 & Design for assembly & $86 \%$ \\
\hline 1.1 & Deployment Subsystems & $79 \%$ \\
\hline 3.1 & Ability to route electrical power and data across assembled joints & $79 \%$ \\
\hline 4.2 & $\begin{array}{l}\text { Ability to disconnect structural, electrical, and fluid connections } \\
\text { without propagating damage to other system components }\end{array}$ & $79 \%$ \\
\hline 10.3 & Modular design & $79 \%$ \\
\hline 10.5 & Design for serviceability & $79 \%$ \\
\hline
\end{tabular}

\section{B. Industry's Estimated Timeline}

For each capability that a company responded that it was pursuing, as discussed in the previous section, the company was asked to estimate when it would have the capability ready. The respondents were given the options of 15 years, 10 years, 5 years, or ready now. The compiled results are presented in Fig. 3Error! Reference source not found. and Table 2Error! Reference source not found.. Figure 3Error! Reference source not found. shows the average estimated development time for each capability, whereas Table 2Error! Reference source not found. shows the distribution of the responses. The purpose of the average is to give the reader a relative assessment of where the sampled respondents as a whole sees themselves in development. Certain capabilities can be pointed out as outliers, such as Capability 1.1 (deployable subsystems), which has the shortest estimated development time, and Capability 2.9 (ability to assemble structures on extraterrestrial surfaces (e.g., Moon, Mars)), which has the longest estimated development time. Capability 5.7 (quantitative performance prediction for autonomous systems) and 7.2 (intelligence for full autonomy) have an estimated development time of more than seven years, whereas the majority of capabilities are estimated to be under six years. While having an average can provide a useful insight, it is worth noting that the average encompasses a breadth of technologies with a range of performance that could be in progress to address a single capability. This figure serves as a gauge of the overall confidence that industry, as represented by the respondents in this assessment, has in the development of each capability.

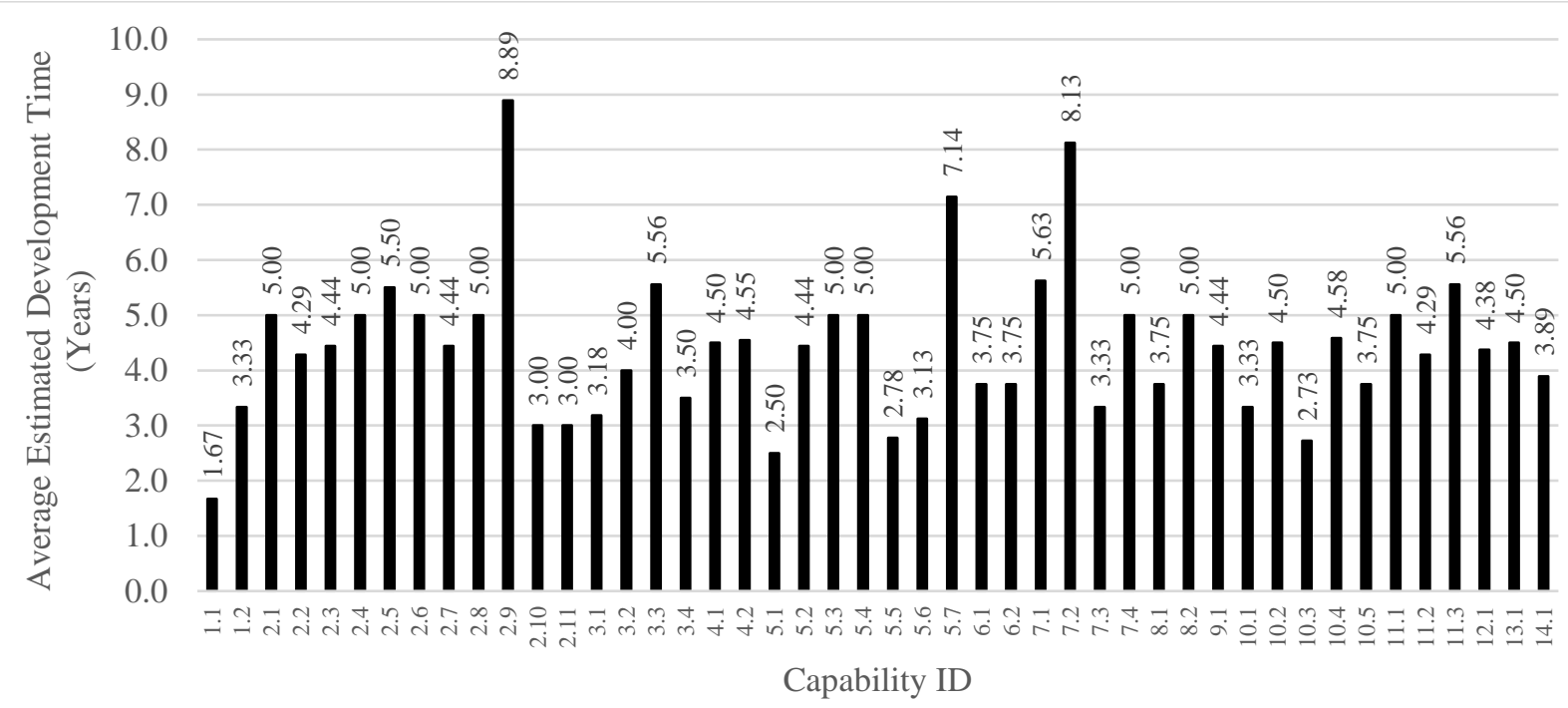

Fig. 3 Industry respondent's confidence level based on the average estimated projected development time per capability.

Looking at the actual distribution of the responses gives a better representation of where industry development was at the time the data was collected (Table 2). The heat map below shows the 46 S\&T iSA capabilities and whether industry respondents are ready with the given capability now, in 5 years, 10 years, or 15 years. The majority of the 
responses are within a 5-year time frame. Therefore, capabilities could be ready sooner than what the average estimated development time indicates. It is worth noting that this does not discuss or show what the corresponding TRL is to each response. The reader would need to investigate the state of the capability for his/her own application.

Table 2 A heat map of the distribution of industry responses to the estimated development time.

\begin{tabular}{|c|c|c|c|c|c|c|c|c|c|}
\hline ID & $\begin{array}{c}\text { Ready } \\
\text { Now }\end{array}$ & $\begin{array}{c}\text { Ready in } \\
5 \text { years }\end{array}$ & $\begin{array}{l}\text { Ready in } \\
10 \text { years }\end{array}$ & $\begin{array}{l}\text { Ready in } \\
15 \text { years }\end{array}$ & ID & $\begin{array}{c}\text { Ready } \\
\text { Now }\end{array}$ & $\begin{array}{l}\text { Ready in } \\
5 \text { years }\end{array}$ & $\begin{array}{l}\text { Ready in } \\
10 \text { years }\end{array}$ & $\begin{array}{l}\text { Ready in } \\
15 \text { years }\end{array}$ \\
\hline 1.1 & $67 \%$ & $33 \%$ & $0 \%$ & $0 \%$ & 5.5 & $44 \%$ & $56 \%$ & $0 \%$ & $0 \%$ \\
\hline 1.2 & $4 \%$ & $1 \%$ & $0 \%$ & $1 \%$ & 5.6 & $38 \%$ & $63 \%$ & $0 \%$ & $0 \%$ \\
\hline 2.1 & $11 \%$ & $78 \%$ & $11 \%$ & $0 \%$ & 5.7 & $14 \%$ & $43 \%$ & $29 \%$ & $14 \%$ \\
\hline 2.2 & $29 \%$ & $57 \%$ & $14 \%$ & $0 \%$ & 6.1 & $50 \%$ & $25 \%$ & $25 \%$ & $0 \%$ \\
\hline 2.3 & $22 \%$ & $67 \%$ & $11 \%$ & $0 \%$ & 6.2 & $50 \%$ & $25 \%$ & $25 \%$ & $0 \%$ \\
\hline 2.4 & $22 \%$ & $56 \%$ & $22 \%$ & $0 \%$ & 7.1 & $38 \%$ & $25 \%$ & $25 \%$ & $13 \%$ \\
\hline 2.5 & $20 \%$ & $50 \%$ & $30 \%$ & $0 \%$ & 7.2 & $25 \%$ & $25 \%$ & $13 \%$ & $38 \%$ \\
\hline 2.6 & $13 \%$ & $75 \%$ & $13 \%$ & $0 \%$ & 7.3 & $44 \%$ & $44 \%$ & $11 \%$ & $0 \%$ \\
\hline 2.7 & $22 \%$ & $67 \%$ & $11 \%$ & $0 \%$ & 7.4 & $25 \%$ & $50 \%$ & $25 \%$ & $0 \%$ \\
\hline 2.8 & $22 \%$ & $56 \%$ & $22 \%$ & $0 \%$ & 8.1 & $25 \%$ & $75 \%$ & $0 \%$ & $0 \%$ \\
\hline 2.9 & $11 \%$ & $22 \%$ & $44 \%$ & $22 \%$ & 8.2 & $25 \%$ & $50 \%$ & $25 \%$ & $0 \%$ \\
\hline 2.10 & $40 \%$ & $60 \%$ & $0 \%$ & $0 \%$ & 9.1 & $33 \%$ & $44 \%$ & $22 \%$ & $0 \%$ \\
\hline 2.11 & $40 \%$ & $60 \%$ & $0 \%$ & $0 \%$ & 10.1 & $44 \%$ & $44 \%$ & $11 \%$ & $0 \%$ \\
\hline 3.1 & $36 \%$ & $64 \%$ & $0 \%$ & $0 \%$ & 10.2 & $40 \%$ & $30 \%$ & $30 \%$ & $0 \%$ \\
\hline 3.2 & $30 \%$ & $60 \%$ & $10 \%$ & $0 \%$ & 10.3 & $64 \%$ & $27 \%$ & $0 \%$ & $9 \%$ \\
\hline 3.3 & $11 \%$ & $67 \%$ & $22 \%$ & $0 \%$ & 10.4 & $33 \%$ & $50 \%$ & $8 \%$ & $8 \%$ \\
\hline 3.4 & $30 \%$ & $70 \%$ & $0 \%$ & $0 \%$ & 10.5 & $42 \%$ & $42 \%$ & $17 \%$ & $0 \%$ \\
\hline 4.1 & $20 \%$ & $70 \%$ & $10 \%$ & $0 \%$ & 11.1 & $43 \%$ & $29 \%$ & $14 \%$ & $14 \%$ \\
\hline 4.2 & $18 \%$ & $73 \%$ & $9 \%$ & $0 \%$ & 11.2 & $43 \%$ & $43 \%$ & $0 \%$ & $14 \%$ \\
\hline 5.1 & $60 \%$ & $30 \%$ & $10 \%$ & $0 \%$ & 11.3 & $33 \%$ & $33 \%$ & $22 \%$ & $11 \%$ \\
\hline 5.2 & $44 \%$ & $22 \%$ & $33 \%$ & $0 \%$ & 12.1 & $50 \%$ & $25 \%$ & $13 \%$ & $13 \%$ \\
\hline 5.3 & $20 \%$ & $60 \%$ & $20 \%$ & $0 \%$ & 13.1 & $30 \%$ & $50 \%$ & $20 \%$ & $0 \%$ \\
\hline 5.4 & $22 \%$ & $56 \%$ & $22 \%$ & $0 \%$ & 14.1 & $33 \%$ & $56 \%$ & $11 \%$ & $0 \%$ \\
\hline
\end{tabular}

\section{Industry's Alignment with Government Activity}

This section compares industry activities in iSA with government activities. Performing a comparative analysis of the industry responses with the government data collected during Phase 2 provides insight into how industry and government can work together to advance iSA capabilities. The analysis indicates the capabilities in which industry respondents are currently planning or pursuing developments that could be provided within 15 years in relation to the 
regions of interagency government collaboration, as defined in Phase 2 by the government needs and investment information [2]. The regions are:

1) High Potential for Collaboration -All three agencies identified the capability need as enabling or supporting one of its operational missions and all identified as investing in the development of the capability need.

2) Potential for Collaboration - At least two agencies identified the capability need as enabling or supporting one of its operational missions and two identified as investing in the development of the capability need.

3) Gaps - Only one agency, or none, identified the capability need as enabling or supporting one of its operational missions and one, or none, identified as investing in the development of the capability need.

4) Low Need - One agency, or none, identified the capability need as enabling or supporting one of its operational missions

5) Some Investment - At least two agencies identified being invested in the capability

6) Low Investment - One agency, or none, identified as being invested in the capability

For each capability, the percentage of industry respondents that stated they were pursuing or planning to pursue that capability is listed in Table 3. This further supports the concept presented earlier of mutual alignment between industry activity and government need, as well as the potential to optimize the collaboration. This is a key element of discussion between the S\&T Partnership and industry. It is recommended that the S\&T Partnership agencies discuss a path forward to collaborate with industry, and identify how various industry iSA technology and capability developments can potentially assist with the S\&T Partnership agencies iSA capability gaps and needs.

Table 3 The range of industry respondents pursuing the iSA capabilities, as mapped within the regions of interagency collaboration (based on the government agency needs and investments)

\begin{tabular}{|l|c|c|}
\hline \multicolumn{1}{|c|}{$\begin{array}{c}\text { Interagency } \\
\text { Government } \\
\text { Collaboration Region }\end{array}$} & $\begin{array}{c}\text { No. of } \\
\text { Capabilities }\end{array}$ & $\begin{array}{c}\text { Percentile Range of } \\
\text { Industry } \\
\text { Respondents } \\
\text { Pursuing } \\
\text { Capabilities }\end{array}$ \\
\hline $\begin{array}{l}\text { LOW NEED, } \\
\text { LOW INVESTMENT }\end{array}$ & 1 & $64 \%$ \\
\hline $\begin{array}{l}\text { LOW NEED, } \\
\text { SOME INVESTMENT }\end{array}$ & 1 & $64 \%$ \\
\hline GAPS & 7 & $21-64 \%$ \\
\hline $\begin{array}{l}\text { POTENTIAL FOR } \\
\text { COLLABORATION }\end{array}$ & 17 & $50-71 \%$ \\
\hline $\begin{array}{l}\text { HIGH POTENTIAL } \\
\text { FOR } \\
\text { COLLABORATION }\end{array}$ & 20 & $43-86 \%$ \\
\hline
\end{tabular}

Table 4 presents the percent of companies from each size category, as defined in this assessment that are pursuing or planning to pursue capabilities in each region. Company size is derived from the self-reported number of employees listed on the company's public professional networking webpage where, small companies were categorized as having 500 employees or less, medium companies were categorized by having $501-1,000$ employees, and large companies were categorized by having 1,001 employees and above. It is noteworthy that, with this grouping of industry respondents by size, the data still showed that small, medium, and large companies from the dataset are pursuing or planning to pursue capabilities from each interagency collaboration region. 
Table 4 The number of companies from each size that work on capabilities in each of the interagency collaboration regions. The column header for size indicates the total number of companies of that size in the dataset (e.g., "out of $15 \%$ " indicates that $15 \%$ of companies in the dataset are medium).

\begin{tabular}{|l|c|c|c|c|}
\hline $\begin{array}{c}\text { Interagency } \\
\text { Government } \\
\begin{array}{c}\text { Collaboration } \\
\text { Region }\end{array}\end{array}$ & $\begin{array}{c}\text { \# of Capabilities } \\
\text { in Each Region }\end{array}$ & $\begin{array}{c}\text { Large } \\
\text { (out of 31\%) }\end{array}$ & $\begin{array}{c}\text { Medium } \\
\text { (out of 15\%) }\end{array}$ & $\begin{array}{c}\text { Small } \\
\text { (out of 54\%) }\end{array}$ \\
\hline $\begin{array}{l}\text { High Potential for } \\
\text { Collaboration }\end{array}$ & 20 & $31 \%$ & $15 \%$ & $46 \%$ \\
\hline $\begin{array}{l}\text { Potential for } \\
\text { Collaboration }\end{array}$ & 17 & $23 \%$ & $15 \%$ & $15 \%$ \\
\hline Gaps & 7 & $23 \%$ & $15 \%$ & $31 \%$ \\
\hline $\begin{array}{l}\text { Low Need, Some } \\
\text { Investment }\end{array}$ & 1 & $23 \%$ & $15 \%$ & $31 \%$ \\
\hline $\begin{array}{l}\text { Low Need, Low } \\
\text { Investment }\end{array}$ & 1 & $23 \%$ & $15 \%$ & $31 \%$ \\
\hline
\end{tabular}

Based on the data in Table 34, Fig. 4 presents the median number of capabilities that companies of each size are pursuing in each collaboration region. Note that the median can only be as high as the number of capabilities in each region. Therefore, the "Gaps" and "Low Need" regions will have fewer capabilities due to the lower number of capabilities in those regions. Fig. 5Error! Reference source not found. presents the percent drop in the median regions (the percent drops between "Gaps" and the "Low Need" regions is not meaningful so they are omitted). The median response for small companies in the dataset drops over 40 percent between "High Potential for Collaboration" and "Potential for Collaboration", whereas the percent drop in the median regions for medium and large companies is insignificant. This indicates that small companies are more likely to pursue capabilities that have high potential for collaboration within the government agencies, whereas medium and large companies are almost equally likely to pursue capabilities that have some potential for collaboration within the government agencies. This drop is significant and could be caused by a number of factors, including:

1) The availability of government funding in those areas - high potential for collaboration means high need and investment by government agencies

2) The number of capabilities in "High Potential for Collaboration" region compared to the other regions; and

3) Capabilities in "High Potential for Collaboration" (e.g., robotic assembly with joining, design for assembly) could be more generic than others (e.g., standard protocols and ports to accommodate visiting vehicles and communication traffic)

The median number of capabilities pursued by small, medium, and large companies drops approximately 60 percent from "Potential for Collaboration" to "Gaps" regions. This is primarily due to the reduction in the number of capabilities in the "Gaps" region compared to the "Potential for Collaboration" region. 


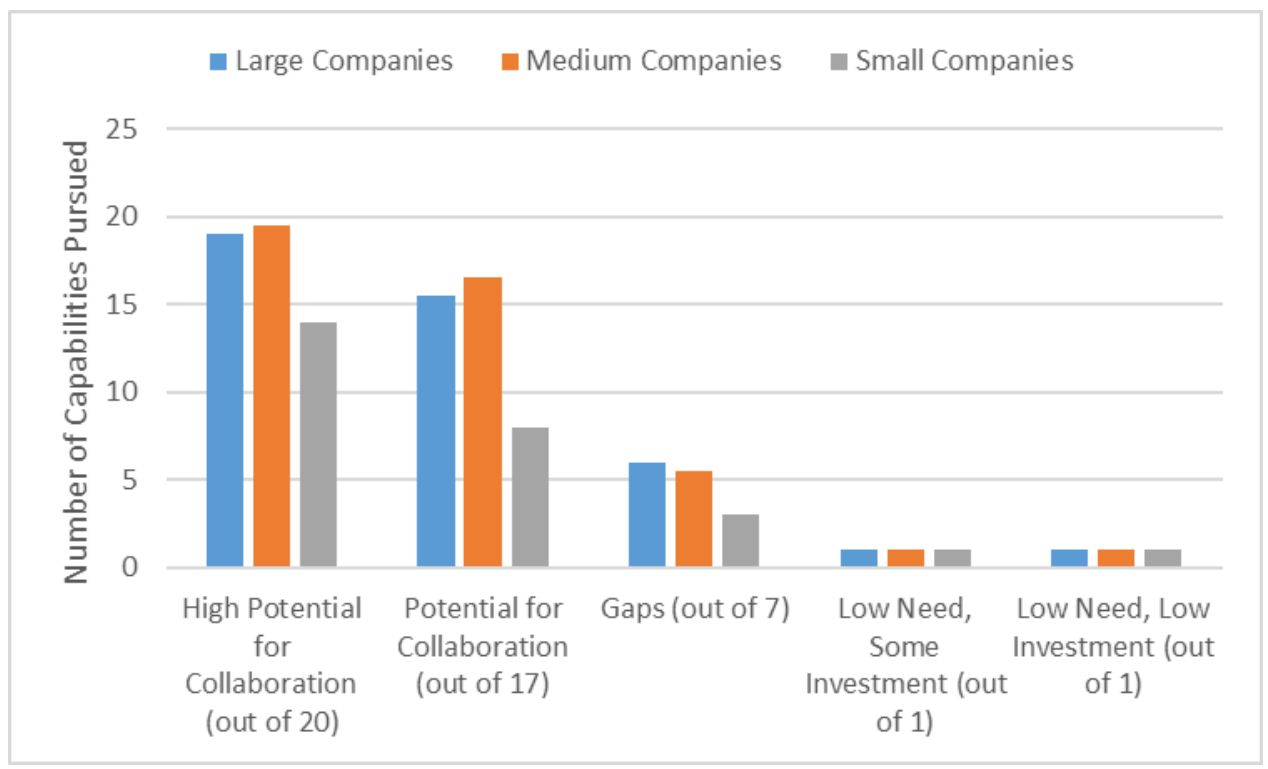

Fig. 4 Median number of capabilities being pursued by companies of different size in each interagency collaboration region.

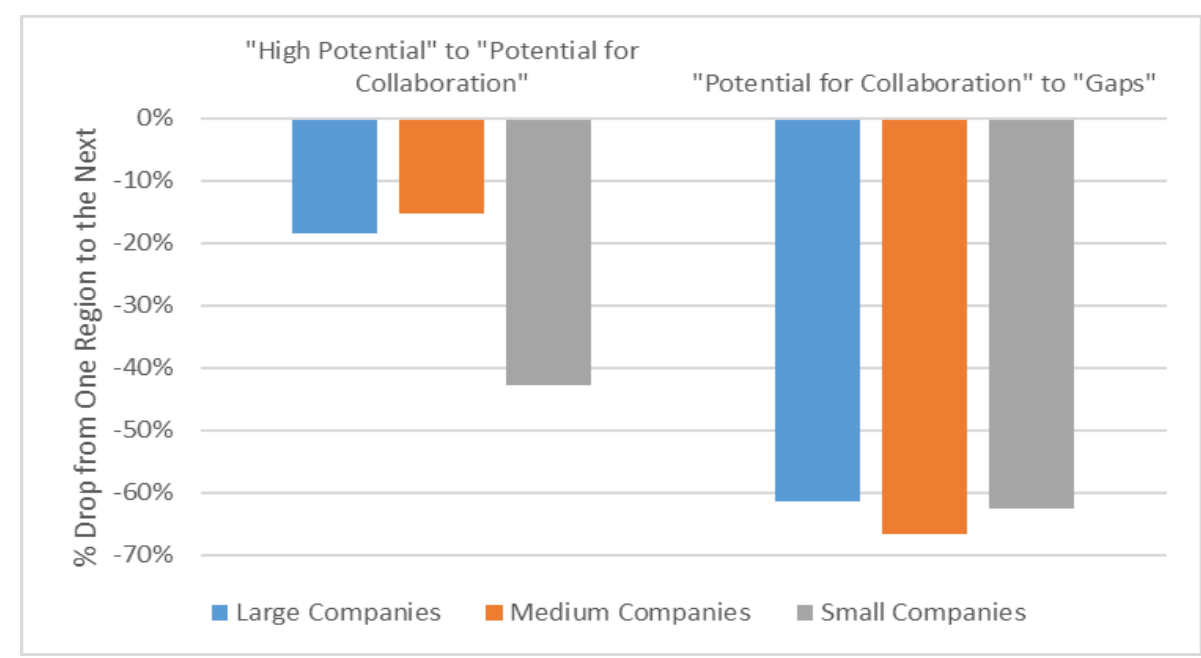

Fig. 5 Percentile drop in median number of capabilities being pursued by companies of different size from one interagency collaboration region to another.

A metric that can reveal the number of companies interested in certain capabilities or capability areas is "volume." In this context, volume is defined as the number of companies in the dataset that are pursuing or planning to pursue a given capability. For example, if three companies are pursuing capability A and seven companies are pursuing capability B, capability B has a larger "volume of work" than capability A. The percentage of total volume across all capabilities by company size is presented in Fig. 6Error! Reference source not found.. Total volume provides an indication of all the ongoing and planned activities (within the dataset) across all of the capabilities. It is noteworthy that the small companies contribute to a substantial amount (45 percent) of the total volume. The larger number of small companies in the dataset compensates for the fact that each small company may be pursuing fewer, more focused capabilities than the medium or large companies. 


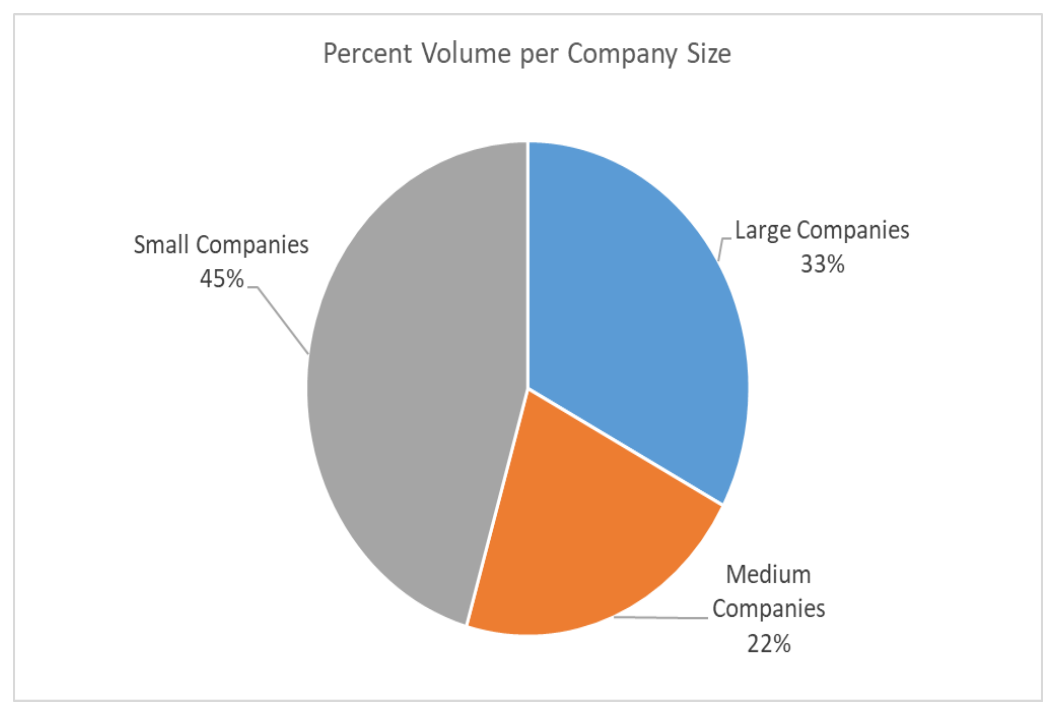

Fig. 6 Distribution of total volume of industry respondents' iSA activity across company size. This distribution approximately holds for each interagency collaboration region.

\section{Industry's Challenges and Barriers}

One of the objectives of this analysis was to identify industry's challenges and barriers for iSA and what potential solutions could alleviate these obstacles. This section gives an overview of the data collection, data process, results, and industry's perspective and recommendations on potential solutions to increase industry activity in the iSA market.

\section{A. Data Collection}

Two questions were asked for companies to identify a challenge or barrier (if any) for a given aspect of iSA:

1) "What are the challenges or barriers to developing this market that an industry/government partnership might overcome?"

2) "What challenges or barriers to the infusion of in-space assembly capabilities in future operational systems do you foresee?"

\section{B. Data Processing}

The Facilitation and Analysis Team collected 79 different challenges for iSA from the participating commercial companies. Challenges were binned into team-defined categories. To determine the category for an iSA challenge, the S\&T team reviewed the given challenge from a company, and based the context of the company and market area, categorized the overarching meaning of the challenge. The challenges were categorized into the following ten themes:

Lack of Business Case - The need for a reason, or justification, for doing a proposed project, mission, or demonstration for in-space assembly (iSA).

Technical Immaturity - Technology that has not been tested or proven to be reliable in a space mission scenario. Technology Readiness Level (TRL) below nine (where within the NASA TRL definitions, TRL nine is categorized as technology that has been "flight proven" during a successful mission).

Lack of an On-Orbit Demonstration Platform - The need for a platform, in space, which allows commercial, academia, and government agencies to test their technology on a space-platform to enhance pre-mature technologies, and advance the Technology Readiness Level through in-space demonstrations.

Lack of Standards - The need for a global rule or definition approved by an authoritative agency to set a specific benchmark for a given technology or capability.

Lack of Collaboration through Public/Private Partnership (PPP) - The need for cooperation with a public company interested in iSA and government agencies participating in iSA, for the purpose of fulfilling an iSA mission or technology demonstration.

Prohibitive Cost - The difficulty of overcoming some financial barrier, due to either restrictions financially, and/or excessively high launch prices. 
Verification \& Validation $(\mathbf{V} \& \mathbf{V})$ - The need to check that a system meets all requirements and specifications in order to fulfill a desired mission.

Technical Risk - A loss arising from the design, engineering, assembly, manufacturing, and/or technology procedures.

Lack of Operational Maturity (Ops) - The need for iSA technology to have the capability to avoid an unforeseen hurdle or encounter occurring real-time during a particular mission or demonstration.

Space Debris Mitigation - The task of reducing the natural (meteoroid) and artificial (man-made) particles from lowEarth orbit.

\section{Technical Findings per Market}

Table 5Error! Reference source not found. shows a breakout of the challenges (by percentages) identified by the applicable companies in each iSA market. The top row shows the challenges that were identified for a given market. The leftmost column represents the market areas that identified the challenges. The percentages represent the frequency companies within the market identified a given challenge.

Table 5 S\&T Industry Open Forum Commercial Space Companies iSA Challenges \& Barriers.

\begin{tabular}{|l|l|l|l|l|l|l|l|l|l|l|}
\hline & $\begin{array}{c}\text { Business } \\
\text { Case }\end{array}$ & $\begin{array}{c}\text { Tech. } \\
\text { Immaturity }\end{array}$ & $\begin{array}{c}\text { Demo } \\
\text { Platform }\end{array}$ & Standards & PPP & Cost & V\&V & Risk & Ops & $\begin{array}{c}\text { Space } \\
\text { Debris }\end{array}$ \\
\hline $\begin{array}{l}\text { Satellites \& } \\
\text { Space Structures }\end{array}$ & $27 \%$ & $22 \%$ & $20 \%$ & $9 \%$ & $7 \%$ & $7 \%$ & $4 \%$ & $2 \%$ & $2 \%$ & $0 \%$ \\
\hline $\begin{array}{l}\text { Satellite } \\
\text { Servicing }\end{array}$ & $30 \%$ & $26 \%$ & $15 \%$ & $7 \%$ & $4 \%$ & $7 \%$ & $4 \%$ & $7 \%$ & $0 \%$ & $0 \%$ \\
\hline Robotics & $16 \%$ & $29 \%$ & $10 \%$ & $16 \%$ & $6 \%$ & $10 \%$ & $0 \%$ & $10 \%$ & $0 \%$ & $3 \%$ \\
\hline $\begin{array}{l}\text { Satellite } \\
\text { Manufacturing }\end{array}$ & $16 \%$ & $31 \%$ & $16 \%$ & $9 \%$ & $4 \%$ & $9 \%$ & $7 \%$ & $7 \%$ & $0 \%$ & $1 \%$ \\
\hline Interfaces & $29 \%$ & $29 \%$ & $7 \%$ & $21 \%$ & $0 \%$ & $0 \%$ & $0 \%$ & $7 \%$ & $0 \%$ & $7 \%$ \\
\hline $\begin{array}{l}\text { Additive } \\
\text { Manufacturing }\end{array}$ & $18 \%$ & $23 \%$ & $9 \%$ & $14 \%$ & $14 \%$ & $9 \%$ & $5 \%$ & $9 \%$ & $0 \%$ & $0 \%$ \\
\hline $\begin{array}{l}\text { Large } \\
\text { Telescopes }\end{array}$ & $50 \%$ & $0 \%$ & $14 \%$ & $7 \%$ & $7 \%$ & $14 \%$ & $0 \%$ & $0 \%$ & $7 \%$ & $0 \%$ \\
\hline Software & $0 \%$ & $0 \%$ & $20 \%$ & $20 \%$ & $20 \%$ & $20 \%$ & $0 \%$ & $20 \%$ & $0 \%$ & 0 \\
\hline
\end{tabular}

On average the four most frequent challenges mentioned were: Technical Immaturity, Business Case, Demonstration Platform, and Standards. The largest hurdles industry respondents discussed were the fact that there is currently a chicken-and-egg paradigm permeating all four of these hurdles and challenges, especially acquiring a business case for iSA. That is, technology for iSA is not available because the market is not available, and the market is not there because the technology is not where it needs to be. The lack of flight opportunities and an available platform makes raising the TRL for companies extremely difficult. Furthermore, indirectly due to a lack of collaboration, standards have not been established, making public-private and private-private collaborations challenging. With the high cost and risks required to venture into this developing market, commercial companies have been slow to enter the field of iSA.

\section{Industry's Recommendations:}

1. Recommendation to $S \& T$ Partnership agencies to assist industry with current iSA challenges:

Industry respondents (87\%) identified technical immaturity, lack of standards (73\%), lack of business case (67\%), and the lack of a persistent on-orbit demonstration platform (67\%) as challenges or barriers for their companies' advancement of iSA. Developing a persistent demonstration platform (and partnerships through this platform as well) could potentially allow the government and industry to:

- Establish an initial testbed to prove a variety of iSA capabilities, proving initial iSA capabilities and testing unproven capabilities, and raising technology TRL's in a space environment.

- Have the opportunity to establish a universal set of standards, advance technologies, in-space robotics, verification and validation, advance plug and play capabilities, and reducing technical risk threshold.

- Give companies a reason to develop iSA technology and launch more demonstration flights, consequently advancing the current iSA market and technologies. 


\section{Government/Industry Partnership Vision, Recommendation:}

Industry respondents (53\%) identified a public-private partnership as a key solution for their company to advance iSA capabilities and technologies. This stems from current limitations due to cost restrictions, lack of flight opportunities, or limited technology, and lack of a business case. Commercial companies believe that assistance from government agencies would be a major factor in addressing these barriers and making iSA become a reality. If the U.S. Government were to provide a continuous joint effort with industry, the respondents thought that some of the risk associated with cost could be relieved, and that the flight opportunities required to raise the TRL for their technologies and a business case in which companies could invest resources would be provided. Several companies noted that the time to develop technologies could be significantly expedited with government funding and support.

As a potential resolution, government agencies interested in partnering with commercial companies should consider federal partnerships such as: Independent Research \& Development (IRAD), Small Business Innovation Research (SBIR), Rapid Innovation Fund (RIF), Commercialization Readiness Programs (CRP), Advanced Technology Program (ATP), DOD Defense Innovation Unit (DIU), or a proposal solicitation to address the earlystage funding requirements faced by commercial companies engaged in the iSA market.

\section{Future Collaboration}

This section focuses on understanding what industry envisions for future collaboration and how industry could contribute and benefit from government efforts. Based off the industry respondents responses, this analysis was done by determining who should lead a specific capability, and one example of how industry's efforts could improve government platforms.

\section{A. Industry's Perspective on Collaboration}

For each capability, companies were asked what they perceived as the best roles for government and industry in development of each capability. The three options were:

1) By industry, then procured as a service by government (government follow)

2) By government then transferred to industry (government lead)

3) By a joint government-industry development (partnership)

The results are compiled in Fig. 7. Overall, industry respondents stated that they want to participate in the development of all capabilities to a degree. The majority of responses stated there should be a joint governmentindustry partnership, with a significant number of responses also expressing industry should lead the development. For all capabilities, the majority of responses stated that development should be a joint venture, except for the following four capabilities, which received a majority of responses stating that industry should lead their development: 1.1 (deployment subsystems), 1.2 (inflatable components), 7.3 (fail-safe modes of behavior on failure detection), and 7.4 (multi-agent autonomy (distributed situation assessment \& coordinated control)). This is surmised to be specifically due to vested interest and current development in these four capabilities. Ultimately, the respondents in this assessment indicated that industry is interested in collaborating and in investing resources. 


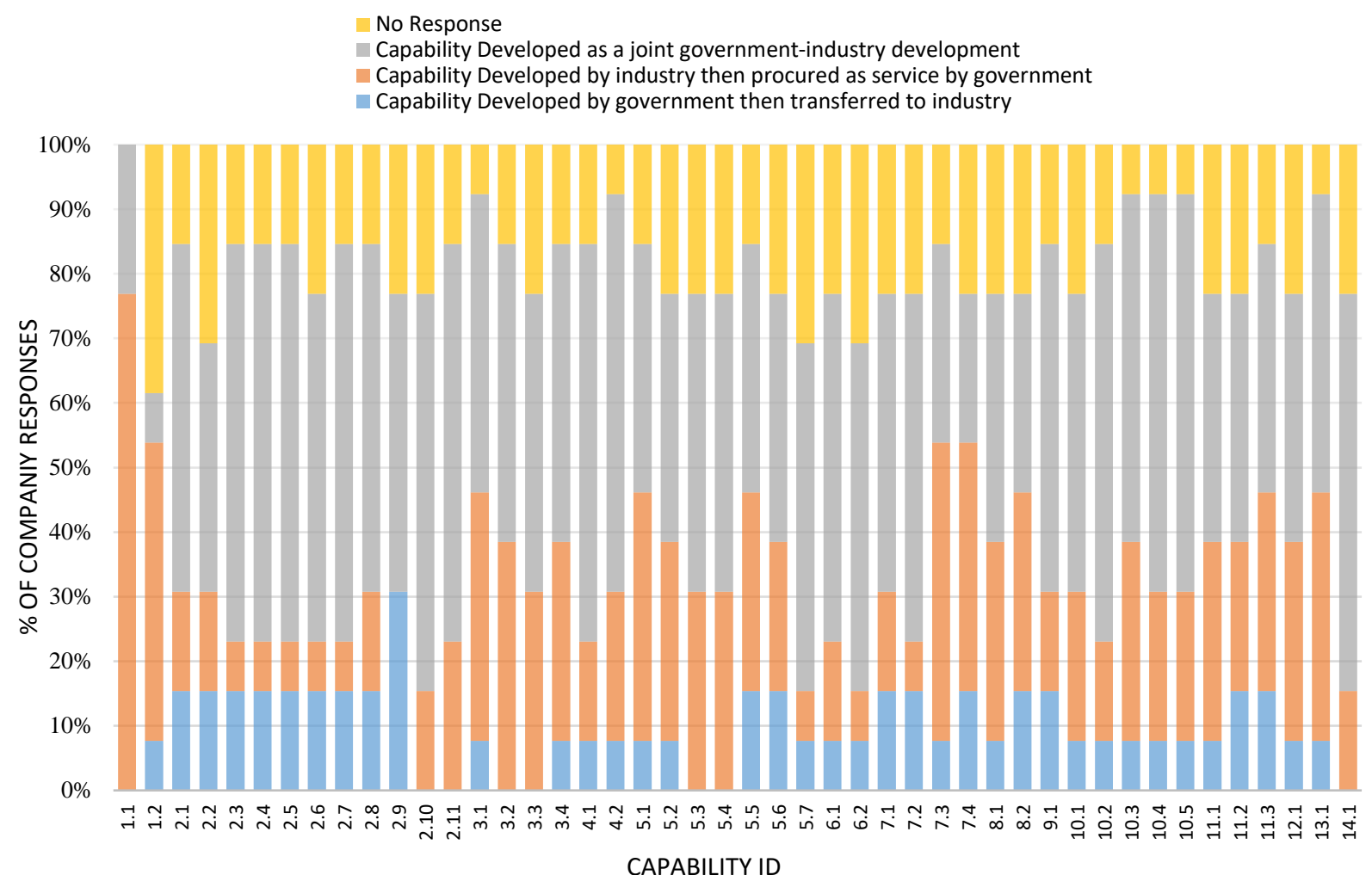

Fig. 7 Industry's perspective on collaboration and who should lead capability development.

\section{B. Industry Efforts with Government Platforms}

During Phase 2, five notional government platforms were analyzed to determine if there were synergies between industry efforts and government platforms' capability accommodations [3]. Each platform was assessed on whether it supported or could support the 46 iSA capabilities identified by the S\&T Partnership, and if so, how much effort would be required to currently support or to add a capability. The results used figures of merit based on modification costs to assess the platforms' ability to provide each of the 46 capabilities and how much of an impact it would have on the program: No Impact, Minor Impact, Significant Impact, Major Impact, or if the platform could not support the capability at all.

To understand how industry could collaborate and assist government programs, time estimates were assigned to the level of impact definitions defined to the platform (Error! Reference source not found.Error! Reference source not found.). The estimated time required for the government to update the platform was then compared to the commercial estimated development time.

Table 6 Platform definition and estimated development impact.

\begin{tabular}{ll}
\hline Platform Definition & Impact Estimated Time (Years) \\
\hline No Impact & 0 \\
Minor & $<1$ \\
Significant & $<5$ \\
Major & $<15$ \\
\hline
\end{tabular}

The figures below show the comparison between maximum time estimated to update the theoretical government platform with a given capability and the estimated development time of a given capability from industry respondents. Capabilities listed without a yellow bar indicate that the platform either: can already support the capability, cannot support the capability at all, or was not mapped to a platform definition. For any of those cases, industry would not be able to collaborate on that particular capability for that platform. Capabilities listed with a yellow bar indicate the platform's ability to add that capability on that platform, in terms of time, using the impact levels shown in Error! Reference source not found.Error! Reference source not found.. If at least one company estimated less than or 
equal to the maximum time to improve the platform, it indicates industry could potentially support adding that capability to a platform. This collaboration could be either developing the capability on the platform jointly with government or potentially developing it sooner than the government and collaborating on integrating the capability to the platform. It is important to note that for companies that responded that they have the capability ready now, considerations need to include the approximate year it would take to integrate and verify with a final space demonstration. Additionally, any opportunities for collaboration on a capability should consider the following in the decision to have an industry add a capability: (1) lifetime of the platform; (2) interface compatibility (H/W, electrical); (3) accommodations for power, data, size, mass, stability; (4) flight opportunity to the platform location; (5) cost; and (6) programmatic impact.

An example of the analysis that could be done to determine collaboration opportunities on the government technology demonstration platforms is shown in Fig. 8. Notional platform A has 20 capabilities that would require minor or significant effort to add those capabilities. In this example, industry respondents (indicated by the blue dot) indicated that they could develop each of the 20 capabilities within the same time frame or sooner. This type of analysis could be used to identify significant opportunities to leverage enhancements on government platforms to encompass more of the S\&T capabilities if there were increased collaboration between industry and government.

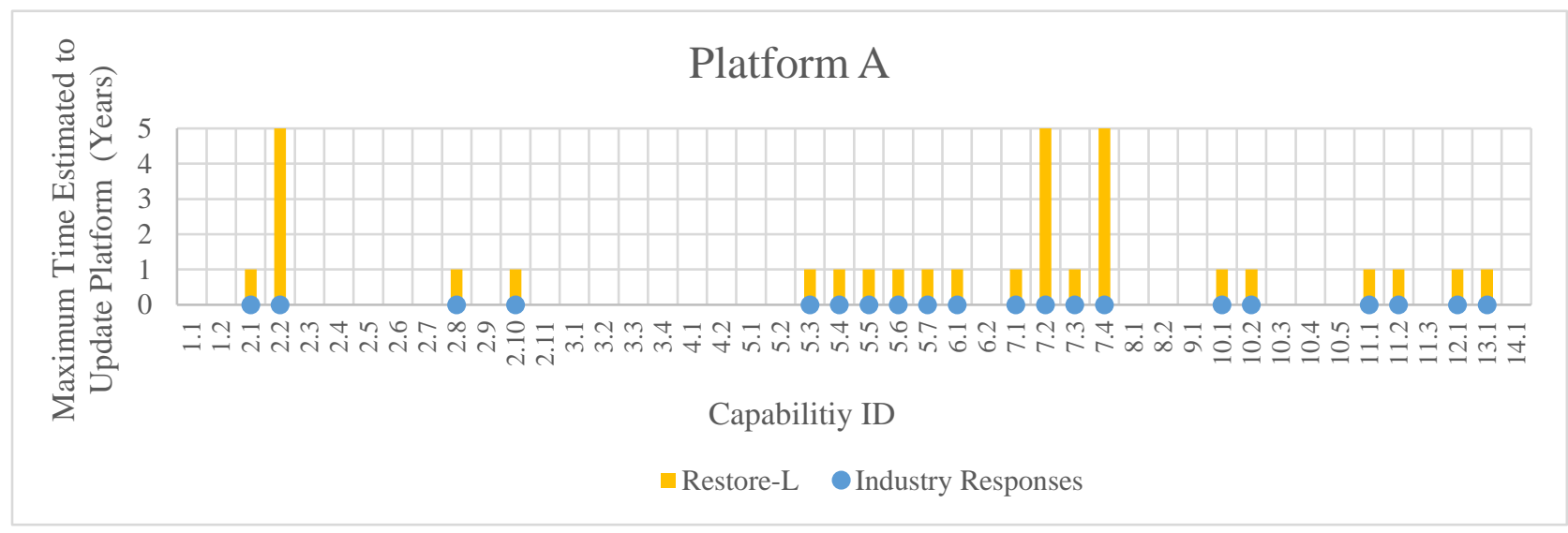

Fig. 8. Notional Platform A analysis identifying which capabilities can be aggregated to the platform and the estimated time frame government and industry could provide those capabilities.

\section{Conclusion}

The S\&T Facilitation and Analysis Team concludes there is a strong interest by industry respondents to collaborate with government agencies for the assistance to facilitate iSA. The analysis shows that all commercial respondents from the Industry Open Forum/TIM-3 are pursuing or planning to pursue capabilities in all regions of government collaboration, especially smaller companies (see Fig. 6) that are responsible for contributing to $45 \%$ of current iSA activities, whereas medium and large companies combined contributed to 55\% of current iSA activities.

Moving forward, the government agencies within the S\&T Partnership should continue cross-agency communication, collaboration, and strategizing on technical solutions to common needs. Agencies should also maintain awareness of each agency's space science and technology investments to reduce duplication and identify areas worthy of collaboration. Lastly, agencies should identify impediments for collaboration and formulate solutions. Most importantly, government agencies in the S\&T Partnership should begin coordinating an interagency planallowing commercial companies' technology developments to be funded by government through SBIRs, CRPs, RIFs, etc. Similarly, partnerships between government agencies with common technological needs for iSA offer efficiencies through cost sharing, elimination of duplication, reduction in overhead costs, pooling of resources, and the exchange of expertise and knowledge.

In addition, industry respondents identified a need for assistance with the development of their technologies and a pervasive persistent platform companies can launch to on orbit to test their developing technologies and capabilities, reducing the technical risk to these developing technologies. The commercial respondents stated they have Technology Demonstration Missions (TDMs), SBIRs, and government operational missions already tied into their funding. This indicates that industry is developing the iSA technologies and capabilities that the U.S. Government is requesting. At least $53 \%$ of the commercial companies surveyed indicated that they were collaborating with the 
government for their iSA technology developments and activities. Thus, government is a strong funding source for the majority of the industry respondents and their iSA technologies, capabilities, and flight opportunities. Government is the main funding source for these companies and their iSA technologies, capabilities, and flights. If the U.S. Government does not continue to help fund these efforts, it is likely that the commercial companies currently involved with iSA technologies may potentially stop production due to little, if any, return on investment. Currently, the cost to develop, test, and mature a technology over time is not financially feasible for any of the commercial company respondents within the iSA market. Yet, over time, as the market grows, and an iSA commodity market develops, these commercial companies may eventually have a strong business case and return on investment to pursue iSA technologies and capabilities with very little, if any, government assistance.

Overall, the positive benefits for iSA greatly outweigh the current challenges and barriers. Over time, iSA has the potential to produce advantages, such as: limiting technical risks, reducing space craft costs, improving performance, as well as enabling new spacecraft dimensions, masses, or configurations that're currently unavailable. Furthermore, iSA has the potential to improve cost-effectiveness through reusability, and allow for a gradual increase of iSA capabilities through upgrades in operation. Through stronger collaborations between industry and government, there are significant opportunities to improve on the existing government platforms to encompass more of the S\&T capabilities and develop a persistent platform in define GEO to allow commercial companies to test their technologies. By doing so, a set of iSA standards and a space economy will begin to be develop, allowing for space technologies to be assessed as a commodity, and growing a healthy government and industry space economy.

\section{Appendix}

Table 7 iSA Capabilities.

\begin{tabular}{|c|l|}
\hline $\begin{array}{c}\text { Capability } \\
\text { ID }\end{array}$ & \\
\hline 1.1 & Deployment Subsystems \\
\hline 1.2 & Inflatable components \\
\hline 2.1 & Robotic assembly with joining \\
\hline 2.2 & Long-reach manipulation \\
\hline 2.3 & Ability to assemble low mass structures \\
\hline 2.4 & Ability to assemble high strength structures \\
\hline 2.5 & Ability to assemble high stiffness structures \\
\hline 2.6 & Ability to assemble structures with micro-stable joints \\
\hline 2.7 & Ability to assemble structures with high dimensional stability \\
\hline 2.8 & Ability to assemble structures with near isothermal control \\
\hline 2.9 & Ability to assemble structures on extraterrestrial surfaces (e.g., Moon, Mars) \\
\hline 2.10 & Ability to deploy hybrid assembly and in-space fabrication processes such as additive manufacturing \\
\hline 2.11 & Provide conductive heat transfer across assembled joints \\
\hline 3.1 & Ability to route electrical power and data across assembled joints \\
\hline 3.2 & Ability to route coaxial cables across joints \\
\hline 3.3 & Ability to route fiber optical conductors across joints \\
\hline 3.4 & Ability to route fluids across joints \\
\hline 4.1 & Ability to reversibly assemble structural, electrical, and fluid connections \\
\hline 4.2 & $\begin{array}{l}\text { Ability to disconnect structural, electrical, and fluid connections without propagating damage to } \\
\text { other system components }\end{array}$ \\
\hline 5.1 & Means of verifying the continuity of interface connections / disconnections \\
\hline 5.2 & Sensors to accurately and precisely measure the quality of the build-up in progress \\
\hline 5.3 & Sensors to accurately and precisely measure the as-built configuration \\
\hline 5.4 & $\begin{array}{l}\text { Sensors to detect failures and/or unacceptable quality of the assembly process after it has been } \\
\text { completed }\end{array}$ \\
\hline 5.5 & Modeling and simulation for verification and validation \\
\hline 5.6 & Modeling and simulation for assembly sequencing / planning \\
\hline 5.7 & Quantitative performance prediction for autonomous systems \\
\hline 6.1 & Standard protocols and ports to accommodate visiting vehicles and communication traffic \\
\hline 6.2 & $\begin{array}{l}\text { Standard but secure communication protocols to accommodate interaction with other (TBD) } \\
\text { associated systems }\end{array}$ \\
\hline & \\
\hline
\end{tabular}




\begin{tabular}{|l|l|}
\hline 7.1 & Intelligence to make stereotyped decisions correctly without human input \\
\hline 7.2 & Intelligence for full autonomy \\
\hline 7.3 & Fail-safe modes of behavior on failure detection \\
\hline 7.4 & Multi-agent autonomy (distributed situation assessment \& coordinated control) \\
\hline 8.1 & Jigging and joining processes capable of achieving a high level of precision open-loop \\
\hline 8.2 & $\begin{array}{l}\text { Known precision limits of any and all assembly agent elements across the assembly site's } \\
\text { environmental envelope }\end{array}$ \\
\hline 9.1 & Tools and approaches to alter a build-up in progress to correct build up errors \\
\hline 10.1 & Tools and component parts capable of accommodating a continuous spectrum of design options \\
\hline 10.2 & $\begin{array}{l}\text { Assembly agent geometries, systems, and tools that do not preclude dimensional or mass growth of } \\
\text { the client system }\end{array}$ \\
\hline 10.3 & Modular design \\
\hline 10.4 & Design for assembly \\
\hline 10.5 & Design for serviceability \\
\hline 11.1 & Ability to accommodate structural members with active length control \\
\hline 11.2 & Ability to accommodate power and data control interfaces associated with active structural members \\
\hline 11.3 & Ability to accommodate TBD sensors for length and/or structural geometry \\
\hline 12.1 & Ability to accommodate passive vibration damping \\
\hline 13.1 & $\begin{array}{l}\text { A limited number of standard mechanical, electrical, thermal, and fluid connection approaches with } \\
\text { well-characterized properties. }\end{array}$ \\
\hline 14.1 & Soft docking/berthing of modules \\
\hline
\end{tabular}

\section{Acknowledgements}

The authors acknowledge the invaluable efforts by contributors at NASA and the other government agency partners in the S\&T Partnership Forum for ensuring this analysis was productive and enlightening and for the technical and editorial reviews of this paper. We thank James A. Dempsey, Robert W. Moses, and Trevor Jackson at NASA Langley Research Center as part of the Facilitation and Analysis team for their contributions to the facilitation of the TIM-3 Open Forum and data acquisition. We also give a special thanks to Georgina Crepps for technical editing support of this paper.

\section{References}

[1] Williams, P. A., Dempsey, J. A., Hamill, D., Rodgers, E., Mullins, C., Gresham, E., and Downs, S., "Space Science and Technology Partnership Forum: Value Proposition, Strategic Framework, and Capability Needs for In-Space Assembly," AIAA Paper 2018-5140, September 2018.Arney, D. C., Williams P. A., Dempsey J. A., Rodgers E., Bozak K., Burley C., Long D., and Husain R., "Space Science and Technology Partnership Forum: In-Space Assembly Data Collection and Analysis," AIAA Paper 2018-5308, September 2018.

[3] Hamill, D. L., Jefferies, S. A., Stillwagen, F. H., Moses, R. W., Mullins, C., and Gresham, E., "Space Science and Technology Partnership Forum: Analysis for a Joint Demonstration of High Priority, In-Space Assembly Technology,” AIAA Paper 2018-5307, September 2018. 